\title{
Meta-Analyses to Investigate Gene-Environment Interactions in Neuroepidemiology
}

\author{
I.A.F. van der Mei P. Otahal S. Simpson Jr. B. Taylor T. Winzenberg
}

Menzies Research Institute Tasmania, University of Tasmania, Hobart, Tas., Australia

\section{Key Words}

Neurological disease $\cdot$ Gene $\cdot$ Environment $\cdot$ Meta-analysis

\begin{abstract}
Background: Most chronic neurological diseases are caused by a combination of multiple genetic and environmental factors. Increasingly, gene-environment interactions (GxE) are being examined, providing opportunities to combine studies systematically using meta-analysis. Methods: Systematic review of the literature on how to examine GxE using observational study designs, and how to conduct a meta-analysis of studies on GxE. Results: Most methods and challenges related to a standard meta-analysis apply to a GxE meta-analysis. There are, however, some substantive differences. With $\mathrm{GxE}$, there is the capability of using a case-only design. Research on GxE interactions may be more prone to publication bias, since interactions are usually not the primary hypothesis and only 'exciting' significant GxE findings are reported out of a range of secondary analyses. In disease aetiology research, there has been debate whether to measure interaction on a multiplicative or additive scale. There are some significant challenges associated with measuring interaction on an additive scale, and thus the uptake of the measures of additive interaction has been limited. As a result, the methods of analysing interaction have been less consistent and reporting has been highly variable. We suggest using the STROBE/ STREGA reporting guidelines to allow evaluation of interaction on both scales. Conclusions: We identified a number of
\end{abstract}

differences of a GxE meta-analysis over a standard meta-analysis. Awareness of these issues is important. Using established reporting guidelines for GxE studies is recommended. The development of consortia for neurological disorders that include both genetic and environmental data might offer benefits for GxE meta-analyses in the future.

(c) 2013 S. Karger AG, Base

\section{Aim of This Review}

This review aims to describe how to examine geneenvironment interactions ( $\mathrm{GxE}$ ) using observational study designs, and how to conduct a meta-analysis of $\mathrm{GxE}$ studies.

\section{Why Examine GxE?}

Many, although not all, chronic neurological diseases are multifactorial, where the disease is caused by multiple genetic and environmental factors. While epidemiologists have traditionally focused on environmental factors and geneticists on genetic markers that may be associated with disease, these researchers are now starting to work together to examine GxE. This is a step forward, since in many circumstances genes and environmental factors will not produce disease in isolation, so it makes sense to examine the combined effect of both.

\section{KARGER}

E-Mail karger@karger.com

www.karger.com/ned
(C) 2013 S. Karger AG, Base

0251-5350/14/0421-0039\$39.50/0
I.A.F. van der Me

Menzies Research Institute Tasmania

Private Bag 23

Hobart, TAS 7001 (Australia)

E-Mail ingrid.vanderMei@utas.edu.au 
Table 1. Definitions of genetic terms

\begin{tabular}{|c|c|}
\hline Genotype & $\begin{array}{l}\text { A genotype is an individual's collection of genes. The genotype is expressed when the information encoded in } \\
\text { the genes' DNA is used to make protein and RNA molecules. The expression of the genotype contributes to the } \\
\text { individual's observable traits, called the phenotype. }\end{array}$ \\
\hline Phen & $\begin{array}{l}\text { A phenotype is an individual's observable traits, such as height, eye colour and blood type. The genetic } \\
\text { contribution to the phenotype is called the genotype. Some traits are largely determined by the genotype, while } \\
\text { other traits are largely determined by environmental factors. }\end{array}$ \\
\hline Locus & $\begin{array}{l}\text { A locus is the specific physical location of a gene or other DNA sequence on a chromosome, like a genetic } \\
\text { street address. The plural of locus is 'loci'. }\end{array}$ \\
\hline Allele & $\begin{array}{l}\text { An allele is one of two or more versions of a gene. An individual inherits two alleles for each gene, one from } \\
\text { each parent. If the two alleles are the same, the individual is homozygous for that gene. If the alleles are } \\
\text { different, the individual is heterozygous. Though the term allele was originally used to describe variation } \\
\text { among genes, it now also refers to variation among non-coding DNA sequences. }\end{array}$ \\
\hline Polymorphism & $\begin{array}{l}\text { Polymorphism involves one of two or more variants of a particular DNA sequence. The most common type of } \\
\text { polymorphism involves variation at a single base pair. Polymorphisms can also be much larger in size and } \\
\text { involve long stretches of DNA. }\end{array}$ \\
\hline $\begin{array}{l}\text { Single nucleotide } \\
\text { polymorphism } \\
\text { (SNP) }\end{array}$ & $\begin{array}{l}\text { SNPs (single nucleotide polymorphisms) are a type of polymorphism involving variation of a single base pair. } \\
\text { Scientists are studying how SNPs (pronounced 'snips') in the human genome correlate with disease, drug } \\
\text { response and other phenotypes. }\end{array}$ \\
\hline $\begin{array}{l}\text { Linkage } \\
\text { disequilibrium }\end{array}$ & $\begin{array}{l}\text { The non-random association of alleles at multiple loci. It is the tendency for the alleles of two separate but } \\
\text { already linked loci on the same chromosome to be found together more than would be expected by chance in } \\
\text { the general population. }\end{array}$ \\
\hline $\begin{array}{l}\text { Hardy-Weinberg } \\
\text { Equilibrium (HWE) }\end{array}$ & $\begin{array}{l}\text { The HWE is a principle stating that the genetic variation in a population will remain constant from one } \\
\text { generation to the next in the absence of disturbing factors. When mating is random in a large population with } \\
\text { no disruptive circumstances, the law predicts that both genotype and allele frequencies will remain constant } \\
\text { because they are in equilibrium. The HWE can be disturbed by a number of forces, including mutations, } \\
\text { natural selection, non-random mating, genetic drift and gene flow. }\end{array}$ \\
\hline $\begin{array}{l}\text { Population } \\
\text { tratification }\end{array}$ & $\begin{array}{l}\text { Confounding by ethnicity. Differences between groups of variable ethnic origins that translate into different } \\
\text { allele frequencies. }\end{array}$ \\
\hline
\end{tabular}

Examining GxE is useful for a number of reasons. First, if we estimate only the separate contributions of genes and environment to a disease while ignoring their interactions, we will incorrectly estimate the proportion of disease (the population-attributable risk) explained by genes, the environment, and their joint effects [1]. Second, the demonstration of interactions assists with the process of causal inference. Given that randomised clinical trials are often unfeasible or unethical in studying disease aetiology, causality of the exposure might need to be inferred from other types of studies. Studies that identify $\mathrm{GxE}$ interaction are a welcome addition to this repertoire, since a demonstration of an environmental factor interacting with a gene on its pathway is supportive of a causal effect. Third, identifying GxE provides additional knowledge on the mechanisms by which different factors might mediate their effect and will, together with other areas of research, slowly unravel these complex diseases.
Lastly, understanding $\mathrm{GxE}$ might allow us to improve preventative advice. For example, we have recently shown that the previously observed association between higher vitamin D levels and reduced relapse risk in multiple sclerosis (MS) only occurs in those with particular alleles of two SNPs located in genes (PRKCZ and PRKCH) belonging to the protein kinase $\mathrm{C}$ (PKC) family [2]. If confirmed, this knowledge could potentially be used to tailor vitamin D supplementation advice in people with MS to those with those particular genotypes.

\section{Why Conduct Meta-Analyses of GxE Studies?}

Genetics has its own nomenclature and we refer the reader to definitions of the most common terms used in this report that are given in table 1 . Similar to genetic studies, it is important that $\mathrm{GxE}$ findings are replicated in 
other studies. While in genetics the replication of main effect findings in another dataset is often required within the same manuscript, this is often not logistically feasible for GxE studies as other studies with data on both the genetic and environmental factors of interest may not exist or be difficult to identify. However, the possibility of false-positive $\mathrm{GxE}$ associations is real, particularly when large numbers of single nucleotide polymorphisms (SNPs) are being tested. In addition, different $\mathrm{GxE}$ studies might have findings variable in magnitude or significance due to differences in sample size or methodology, including the measurement of the exposure or genotype, and selection of the study populations.

A meta-analysis combines the results of several independent studies that are considered to be sufficiently similar in methodology to allow this. Ultimately, the primary aim of a GxE meta-analysis is to resolve whether a true interaction is present, but it will also examine the magnitude and pattern of interaction present [3]. Given that the number of studies which examine GxE has substantially increased in recent years, opportunities for meta-analyses are increasing.

\section{How to Examine Interaction?}

In the most general sense, two risk factors are said to interact with one another if the effect of the first factor on disease risk depends on the level of the second factor and vice versa. In MS for example, both high Epstein-Barr virus nuclear antigen (EBNA) IgG levels and smoking are independent risk factors of MS. However, we have shown that the increased risk of MS associated with high EBNA IgG levels was stronger for ever-smokers [OR $=3.9$ (95\% confidence interval 2.7-5.7)] compared to never-smokers [OR $=1.8(1.4-2.3), \mathrm{p}<0.001$ for interaction] [4].

Interaction can be additive or multiplicative, which refers to whether the interaction is assessed on either an additive or multiplicative scale. There is debate about which method is preferable. Most researchers assess interaction by entering a product term into the linear or logistic regression model. In linear regression analysis, the coefficient of the product term for interaction means departure from additivity (i.e. interaction is assessed on an additive scale), whereas in logistic, log-binomial and Cox regression the regression coefficient of the product term estimates departure from multiplicativity, since these models relate exposure(s) and outcome through a logarithmic link, hence on a multiplicative scale. In disease aetiology, where logistic regression models are used, interaction on a multiplicative scale is said to exist if the relative risk of having both factors does not equal the product of the relative risks associated with each factor separately (in the absence of the other factor). While this test is easy to apply, it has been argued that it is more useful biologically to test for interaction on an additive scale [5]. Interaction on the additive scales is said to exist if the excess risk attributable to both factors does not equal the sum of the excess risks caused by each factor separately (in the absence of the other factor).

Additive interaction is measured in cohort studies primarily using risk differences, also known as excess risk due to interaction (ERI) or interaction contrast. In cohort studies, this can be obtained by fitting linear or log-linear risk models. However, the ERI cannot be estimated from case-control studies unless the sampling fractions for cases and controls are known or can be estimated [6]. Alternative measures for interaction on the additive scale have been based on risk ratios. These measures are the relative ERI (RERI), attributable proportion due to interaction (AP) and synergy index (SI) [7].

Importantly, whether interaction is present can depend on whether a multiplicative or additive scale is used. It is therefore proposed that risk estimates for four distinct groups should be presented so that interaction can be assessed on both scales. These groups are: background group (B), those without the genotype and without the exposure; the exposure-only group (E), those with the exposure but without the genotype; the genotype-only group $(G)$, those with the genotype but without the exposure; and the gene and environment group (GE), those with both the genotype and the exposure [8]. The estimates for groups $\mathrm{E}$ and $\mathrm{G}$ are called the environmental main effect and genetic main effect, respectively.

There are challenges to using the additive interaction parameters and the associated uptake of these measures has been limited as a result. Compared to a cohort-study where the absolute baseline risk is known, the interpretation of the additive interaction measures in case-control studies is more difficult. For example, the AP can become negative when antagonistic interaction exists, and this negative proportion cannot be interpreted as the percentage of the observed risk in those with both factors that is due to the additive interaction. In relation to the coding of the genotype and exposure, for multiplicative interaction, when the coding of a variable is reversed, the multiplicative interaction parameter simply becomes the inverse, the power is not altered and neither is the conclusion about whether multiplicative interaction is present. However, for additive interaction, the formulas of the 
three measures are not each symmetrical. Therefore, a different comparison group will result in a different denominator, will provide different magnitudes, $p$ values and confidence intervals, and possibly different interpretations. For additive interaction, the optimal coding scheme is one where estimates of relative risk for group $\mathrm{E}, \mathrm{G}$ and $\mathrm{GE}$ result in values $>1$. However, this might not always result in the preferred public health messages when translating findings to the target audience. In relation to adjustment for confounding, the RERI and AP vary across strata defined by the covariates, whereas SI and the multiplicative interaction parameter do not vary across strata, leaving the SI the measure of choice for additive interaction [6].

\section{Patterns of GxE}

Different patterns of interaction have been proposed [3], depending on whether or not there is a significant environmental main effect, genetic main effect and GxE effect. In the classical synergistic or antagonistic interaction model, one might see a significant environmental main effect and a significant genetic main effect, and the effect of having both factors is significantly higher (synergistic) or lower (antagonistic) than expected under an additive or multiplicative model of no interaction. It is also possible that there is one significant main effect and that the presence of the other factor results in a stronger or weaker effect than expected. Lastly, it is also possible that there are no significant main effects, while having both the exposure and genotype produces a significant risk or protective effect.

\section{Study Designs for GxE Including the Case-Only Design}

While there are some genetic study designs, such as family-based studies, that are capable of analysing GxE using novel methods such as G-estimation, we limit our discussion to epidemiological study designs. All observational study designs, including cross-sectional, case-control and cohort studies can be used to examine GxE.

A specific design to examine $\mathrm{GxE}$ is the case-only design. One can obtain the multiplicative interaction parameter by having cases only. This design allows a gain in power; however, the key assumption is that the distribution of the genetic marker is independent of the exposure in the general population (e.g. controls) [9]. A disadvan- tage is that the environmental and genetic main effects (estimates for group E and G, respectively) as well as additive interaction parameter cannot be obtained from this design [9]. Multiplicative interaction can be estimated from the odds ratio relating exposure and genotype in cases (e.g. odds of having the risk allele among exposed over the odds of having the risk allele among unexposed). Under the independence assumption, this same odds ratio in the controls would be 1 (i.e. no association between exposure and genotype). When the independence assumption holds, the multiplicative interaction estimates are similar for case-only compared case-control studies [10]. While it is not possible to test the independence assumption in an individual study with only cases, a metaanalysis is likely to include studies with data available on controls. In those studies, the independence assumption can be tested.

While it is not common that the assumption does not hold, an association between the genotype and exposure might occur by chance, when the genotype influences behaviour [11], indirectly due to one's knowledge of gene status [12] or due to population stratification [13]. For example, a hypoactivity of the enzyme aldehyde dehydrogenase-2 (involved in ethanol metabolism), due to some genotypes of the gene $A L D H 2$, leads to a build-up of an ethanol metabolite following alcohol consumption, resulting in flushing of the face, nausea and/or headache, which in turn results in a lower alcohol intake [11]. The breast cancer gene BRCA1 may be associated with reduced use of postmenopausal hormone therapy, because a woman with a family history of breast cancer is more likely to carry the BRCA1 mutation and, knowing her family history, may be more likely to avoid postmenopausal hormones [12]. Population stratification (confounding by ethnicity) can yield associations between exposure and genotype since, for any disease which varies strongly with a particular population, any environmental or genetic predictor of disease may also appear to be associated with that population status [13].

\section{Process of Conducting a Meta-Analysis of GxE}

Meta-analysis is the use of statistical methods to summarize the results of independent studies and is performed as part of the process of a systematic review. There is no meta-analysis handbook for observational studies, but the Cochrane Handbook for Systematic Reviews of Interventions is a useful guide [14]. A meta-analysis consists of seven broad steps: van der Mei/Otahal/Simpson/Taylor/ Winzenberg 
(1) define the research question to be addressed, such as the existence and magnitude of GxE;

(2) identify potentially relevant research reports, including unpublished data, using a comprehensive, systematic search strategy;

(3) select the studies to be included in the final meta-analysis using pre-specified inclusion and exclusion criteria;

(4) extract measures of effect size, a description of salient study characteristics and assess study quality based on a predefined protocol;

(5) consider whether it is appropriate, given the clinical and methodological diversity in the identified studies, to proceed to meta-analysis - if it is appropriate to proceed, convert all effect measures to a common metric;

(6) combine the individual study estimates using standard meta-analytic techniques that weight effect measures by an estimate of their precision to obtain pooled effect size and confidence interval; and

(7) carry out analyses to investigate statistical heterogeneity and identify possible modifiers of the effect, and sensitivity analyses to investigate the robustness of the findings to any assumptions or decisions on review methods made in the systematic review process and investigate potential publication bias.

In the next sections we will focus less on generic metaanalyses and instead focus on those issues that are different or particularly relevant in meta-analysis of GxE.

\section{Statistical Methods to Conduct a Meta-Analysis}

The first consideration is to determine the type of meta-analytic model. For GxE studies, which are typically observational, a random-effects model is recommended, since the underlying assumptions that studies are a sample of all possible studies and that studies are typically not functionally equivalent, are more likely to be appropriate. A disadvantage is that random-effects models give relatively more weight to smaller studies than fixed-effect models and as a result are potentially more influenced by publication bias, which is an issue in GxE meta-analyses, as discussed below.

In disease aetiology research, the outcome will often be dichotomous using study designs such as case-control and cohort studies, and logistic or log-binomial regression might be used. Where studies report a measure of interaction, either the multiplicative or additive interaction parameters (RERI, AP and SI), these are pooled by the usual methods of meta-analysis (inverse variance weighting) [14]. Stratum-specific estimates of relative risks presented as two $2 \times 2$ tables or risk ratios for each stratum can also be pooled by calculating the appropriate risk ratio or risk difference for multiplicative test of interaction [15].

For continuous outcomes using a linear regression model, where interaction has been assessed on an additive scale (by inclusion of a multiplicative term), pooling is performed using standard methods of meta-analysis of the interaction coefficient. Where the interaction coefficient is not reported but rather stratified estimates of effect are reported by subgroup, the interaction effect can be calculated using standard statistical methods for the difference between subgroup effects (and pooling of subgroup variances).

Where there is variability in reporting of effects, it is necessary to transform effects to a common metric. Techniques exist for transforming main effects from continuous to dichotomous and back again with certain assumptions [16]; however, there can be complications in converting interaction parameters to make them comparable across different scales. An example of a potential useful method can be found in the example discussed below (fig. 1) [17], where effects are converted to correlations between exposure and outcome for each genotype group, and then the difference of correlations for between genotype groups is calculated to assess interaction.

Meta-analysis of interactions for other effect measures such as hazard ratios or rates follows the standard metaanalytical methodology when studies report interaction coefficients from regression-based modelling. Some of the above methods may also be applied when regression estimates are reported in a stratified manner.

Testing of heterogeneity follows the standard methods used in meta-analysis, including assessment of the Q and $\mathrm{I}^{2}$ statistic. Subgroup analyses and meta-regression can be used to further investigate the causes of heterogeneity [18]. A plot of studies ordered chronologically may reveal whether early studies provide inflated estimates over subsequent studies, also called the 'winners curse' or 'Proteus phenomenon'. Presenting ethnic group-specific estimates can assess the effect of possible population stratification. Publication bias, where authors are more likely to publish a paper if they obtain statistically significant findings, should be evaluated where possible, using a funnel plot and statistical test. A number of tests have been proposed, and the choice depends on characteristics of the meta-analysis [19]. Asymmetry in a funnel plot, however, may be due to publication bias but could also be due to other explanations, including heterogene- 


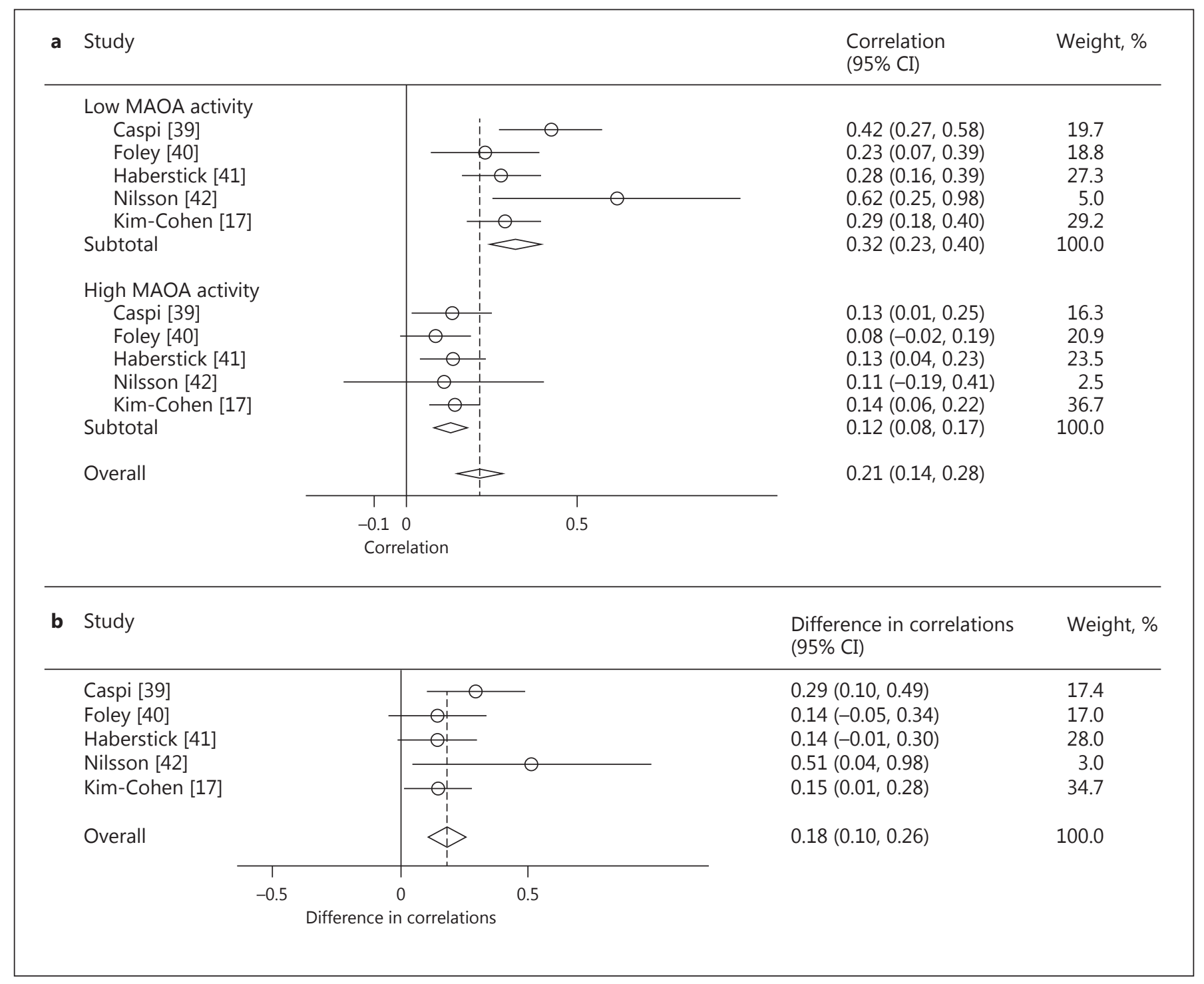

Fig. 1. Meta-analysis pooling results across studies. Summary correlations between measures of childhood maltreatment and mental health are presented separately in the low- versus high-activity MAOA genotype groups. Squares and \% weight indicate the size of each study's contribution to the summary correlations indicated by diamonds. a Forest plot of correlations between childhood mal-

ity (based on methodological quality, selective outcome reporting or small study bias). If publication bias is suspected, the analysis can also be concentrated on larger studies.

Sensitivity analyses are recommended to assess whether the results are robust to the decisions in the methods of the systematic review, for example inclusion criteria, imputation of data, particular assumptions and/ treatment and mental health, as a function of MAOA genotype group in five independent studies. b Forest plot of the interaction effect based on differences in correlations between childhood maltreatment and mental health observed in low- versus high-activity MAOA genotype groups in five independent studies. Reproduced from Kim-Cohen et al. [17].
44

Neuroepidemiology 2014;42:39-49 DOI: $10.1159 / 000355439$ or study quality. From the genetic perspective, it is recommended that the genotype distribution should follow the Hardy-Weinberg equilibrium (HWE) within each study and sensitivity analyses be performed excluding studies where the sample violates HWE [20]. For cohort or cross-sectional studies, the sample of all subjects should be assessed for HWE; in case-control studies only controls should be assessed since it is expected that 
cases may not be in HWE if an association between genotype and disease exists. Deviations from HWE in healthy populations may be a sign of selection bias, population stratification, or even genotyping errors [21]. For meta-analysis on genetic association studies, it is recommended to evaluate the magnitude of departure from HWE in control subjects rather than performing significance tests [21].

\section{Examples of GxE Meta-Analyses}

A review of the literature shows that most GxE metaanalyses are done using dichotomous or categorical exposure variables [22]. Currently, few [23] meta-analyses have used additive interaction parameters for assessment of GxE. Most analyses include a stratified analysis where the effect of the genotype on disease outcome is reported stratified by the exposure, or the effect of the exposure stratified by the genotype, as well as an estimated measure of interaction. A range of outcomes have been used, including dichotomous outcomes (e.g. having an oral cleft [24], colorectal adenoma and colorectal cancer [25], bladder cancer [26], and heavy smoking [27]), categorical outcomes (e.g. amount smoked coded as an ordinal variable [27]) or continuous outcome (e.g. scales of antisocial behaviour [17], and fasting glucose or insulin levels [28]). The genotype ranged from one specific SNP to a genetic risk score based on loci previously associated with the outcome (e.g. number of risk alleles at 16 loci [28]). If a phenotype correlates highly with an SNP, this can also be used. One meta-analysis combined studies using $\mathrm{N}$-acetyltransferase 2 acetylation phenotype and genotype, both providing a good measure of acetylation status and related to the liver's detoxification ability [26]. This was shown to interact with cigarette smoking in causing bladder cancer. The authors used a case-only design and found an interaction OR of $1.3(1.0-1.6)$, suggesting that the association between ever smoking and bladder cancer was 30\% stronger among slow acetylators compared with rapid acetylators [26]. They demonstrated that the independence assumption held among controls by using the studies that had controls available.

Kim-Cohen et al. [17] analysed whether a functional polymorphism in the promoter region of the monoamine oxidase A (MAOA) gene moderated the impact of childhood maltreatment on the risk for developing antisocial behaviour. MAOA has long been recognised for its role in human behaviour, with an aberrant activity in this enzyme negatively affecting the normal flow of neurotrans- mitters and affecting mood and neurological function. The meta-analysis included five studies. The genotype consisted of five variants of the 30-base pair repeat sequence. All studies defined the two most common alleles, 3 repeats, as low activity, and 4 repeats as high activity. There was not complete concordance regarding the classification of some of the rare alleles, but sensitivity analysis showed that this did not influence the results. Both the exposure (childhood maltreatment) and the outcome (mental health problems) are complex to assess and the studies used different and often multiple methods to measure them. Childhood maltreatment was dichotomised into exposed and not exposed, and the outcome was dichotomised into present and not present. Effect sizes were converted into a common metric of correlations and all correlations were $\mathrm{z}$-transformed and their standard errors derived. Figure 1 provides an example of how to present GxE meta-analysis data. Figure 1a shows a forest plot of correlations for each study between childhood maltreatment and mental health grouped by low versus high MAOA activity. Figure $1 \mathrm{~b}$ shows the interaction effect using the differences in the correlations between the $M A O A$ groups in each study. The pooled results showed a significant difference in correlations [0.18 (0.10-0.26), $\mathrm{p}<0.001$; fig. $1 \mathrm{~b}]$, with a stronger correlation between childhood maltreatment and mental health in the low MAOA activity group $[\mathrm{r}=0.32(0.23-0.40)]$ compared to the high MAOA activity group $[\mathrm{r}=0.21(0.14-0.28)$; fig. 1a]. No significant heterogeneity was detected when examining the difference in correlations, using the $x^{2}$ based Q statistic ( $\mathrm{p}=0.44)$ and the $\mathrm{I}^{2}$ statistic $(0.0 \%)$, although there was mild-moderate heterogeneity across studies in the low-activity MOAO group according to the $\mathrm{I}^{2}$ statistic $(36.4 \%)$. Sensitivity analysis included the removal of the first study on this topic and a study with a large effect size. The pooled effect remained significant [difference in correlations $0.15(0.05-0.24), \mathrm{p}=0.002$ ].

Hartz et al. [27] examined the interaction between an SNP in the a5-nicotinic receptor subunit gene CHRNA5 (rs16969968 or its proxy rs105730) and age of smoking onset in predicting subsequent smoking behaviour. The exposure was age at smoking onset $(\leq 16$ years or $>16$ years) and the primary outcome was dichotomous, heavy ( $>20$ cigarettes/day) versus light ( $\leq 10$ cigarettes/day) smoking, omitting those participants with an intermediate level. The SNP was previously shown to be associated with smoking behaviour. There is also a well-established association between early age at onset of smoking and higher risk of heavier smoking and nicotine dependence. The meta-analysis showed that the increased risk be- 
tween early onset smoking and subsequent risk of heavy smoking was stronger among those with an A allele of the SNP [summary OR 1.45 (1.36-1.55)] compared to those with the GG genotype [summary OR 1.27 (1.21-1.33)] (p value for difference in effects 0.01 ). Sensitivity analysis provided similar results when the outcome was coded as a four-level variable using linear regression, or when age of onset was coded differently.

\section{Challenges when Conducting a GxE Meta-Analysis}

The challenges faced when undertaking a GxE metaanalysis are discussed below. While some of these are shared with other meta-analytic studies, we highlight some specific issues related to $\mathrm{GxE}$ meta-analysis.

\section{Lack of Consistency in the Definition and}

Measurement of the Genotype, Environmental

Exposure and Outcome

It is important to set eligibility criteria for meta-analyses that include consideration of which methods of defining and measuring genotype, environmental exposures and outcomes are suitable for inclusion. Specifically for GxE studies, different studies might not exactly report the same genetic variant or report a different genetic model (additive, dominant or recessive). Some environmental exposures can be measured in different ways and the way in which presence or absence of a factor is defined for each measurement method needs to be decided. A recent review concluded that $\mathrm{GxE}$ meta-analyses only seem to be done using dichotomous or categorical environmental variables [22]. With purely continuous exposures, the measures of exposure were too heterogeneous for a quantitative synthesis to be feasible [22]. Some advanced methods of synthesis have been developed to combine information reported in different or incomplete ways using, for example, a Bayesian method. The definition of having a particular disease (yes, no) as an outcome is often relatively straight forward, but can be more complex, as seen in the example from Kim-Cohen et al. [17], where different mental health outcomes were measured.

\section{Lack of Consistency in Study Designs}

The types of studies that might examine GxE are cohort studies, case-control studies, cross-sectional studies and case-only studies. They might produce different results and are likely to contribute to heterogeneity. It is important to limit to, or stratify by, study designs that are appropriate to answer the specific research question.

\section{Bias of Observational Studies}

The individual observational studies will be more or less prone to different types of bias (e.g. selection bias, information bias, confounding) or measurement error. Individual studies should be carefully examined for the likelihood of different types of bias and to investigate whether appropriate adjustments have been made to address potential sources of bias. An assessment of study quality is recommended, although there are currently no well-accepted methods or scales of quality for non-randomised meta-analyses [29] or genetic meta-analyses [20].

With regard to the effect of selection bias on estimates of $\mathrm{GxE}$, it has been shown that for $\mathrm{GxE}$ estimates on the multiplicative scale the estimates are not subject to selection bias when the genotype itself does not influence participation [30]. This applies even when selection is influenced by exposure and disease status, and genotype is associated with either or both of these. Similarly, in a hospital-based case-control study, Wacholder et al. [31] observed that even if exposure or genotype or both are associated with the condition that led to the hospitalisation of control subjects, a departure from multiplicative effects can be estimated without bias. However, this is not the case for departure from additive effects.

Information bias can occur as a consequence of errors in assessing the genotype or exposure. The bias is differential if the measurement error of the genotype or exposure differs by the outcome of interest. Factors that may influence the genotype information include the type and quality of samples, timing of collection, the method used for genotyping, and quality control procedures. To prevent differential misclassification, it is important that research staff are blinded to the outcome of interest, and that the genotyping was performed together, for example, cases and controls. Clayton and McKeigue [32] argue that differential and non-differential misclassification of dichotomous factors tends to underestimate departure from a multiplicative effect, when the genotype and exposure are independent in the source population, and the errors in the assessment of each are independent. The impact of misclassification on departures from additive effects is difficult to predict [33].

Confounding of exposures is a potential problem, the same as it is for exposure-disease associations. Population stratification is a confounding issue for genetic and $\mathrm{GxE}$ studies, where differences between groups with different ethnic origins translate into different allele frequencies. Controversy surrounds the potential importance of population stratification. There is some evidence against a van der Mei/Otahal/Simpson/Taylor/ Winzenberg 
large effect of population stratification [34, 35], although it could be larger under some circumstances (e.g. large sample sizes, rare allele frequencies) [35].

Lack of Consistency in Statistical Analysis Models and Methods for Assessing Interactions

The models used to estimate interaction may differ depending on the outcome. In addition, as mentioned, the method used to assess interaction is a matter of debate. As a result, some studies examine interaction on a multiplicative scale and some on an additive scale, and the conclusion on whether or not interaction is present can simply depend on the scale that is used. Some of the above issues could be overcome by conducting an individual-participant meta-analysis. Although obtaining the data from the individual research groups is the biggest challenge, and it is time-consuming to harmonise the data, the rewards are high. Advantages include use of the same analytical techniques, improved capability to harmonise genotype and exposure data, consistency of control for confounding, increased ability to evaluate alternative genetic models and multiple genes, consistent treatment of subpopulations, and better assessment of bias. Currently, several genetic consortia exist for neurological disorders (e.g. International Multiple Sclerosis Genetic Consortium; International Stroke Genetics Consortium). These strive towards the use of individual participant data and the harmonisation of genetic data. Expansions towards the inclusion of environmental data might offer benefits for GxE metaanalyses in the future.

\section{Lack of Consistency in Reporting of Interactions}

The way GxE interactions are reported varies substantially. In case-control and cohort studies, some only reported $\mathrm{p}$ values for interaction $(\sim 20 \%)$; some provided separate effect estimates and confidence intervals across strata but without meaningful comparison of stratumspecific effect estimates ( 30\%); some provided effect estimates and confidence intervals across strata of the other factor and a p value or statement of statistical significance based on an appropriate test for interaction $(\sim 40 \%)$, and, finally, some provided sufficient information to interpret the interaction on an additive and multiplicative scale $(\sim 10 \%)$, i.e. individual effect estimates and joint effect using 1 reference category, or by presentation of the full model (individual effect estimates and product term) [36].

For individual studies, we recommend using the reporting method suggested by the Strengthening the Reporting of Observational Studies in Epidemiology
(STROBE) statement [37] and its extension for genetic associations studies (STREGA) [38]. It advises a transparent presentation of both main effects and joint effect, each relative to the reference group, allowing evaluation on both the additive and multiplicative scale.

\section{The High Risk of Publication Bias}

Individual studies are prone to publication or reporting bias, whereby authors are more likely to publish a paper if they obtain statistically significant findings or only report those associations reaching significance. This might be even a greater issue for $\mathrm{GxE}$ studies. Generally, larger sample sizes are needed to detect interactions compared to main-effect studies, while studies are often powered for main effects. This might lead to falsenegative associations, and only 'exciting' significant GxE findings being reported out of a range of secondary analyses.

The problem of publication bias is often hard to overcome. The only true test is to compare results from published studies with results from comparable unpublished studies, and these are nearly always unknown. Clinical trial registration systems have been introduced for intervention studies in an effort to address publication bias. This works because scientific journals that are members of the International Committee of Medical Journal Editors require, as a condition of publication, registration in a public trials registry. At present there are no comparable registers for GxE studies and whether this approach would be feasible in genetic epidemiology research is unclear.

\section{Conclusions}

With the increasing number of studies examining $\mathrm{GxE}$, it is likely that the use of GxE meta-analyses will increase in the future. While most methods and challenges related to a standard meta-analysis apply to a GxE metaanalysis, there are substantive differences. Firstly, with GxE there is the capability of using a case-only design. The independence assumption that underlies this method might, however, not always hold. Secondly, research on GxE interactions might be more prone to publication bias because interactions are often not the primary hypothesis and only 'exciting' significant $\mathrm{GxE}$ findings are reported out of a range of secondary analyses. Lastly, in disease aetiology research, there is debate about whether to measure interaction on a multiplicative or additive scale. There are some significant challenges associated 
with measuring interaction on an additive scale and the associated uptake of the measures of additive interaction has been limited. As a result, the methods of analysing interaction have been less consistent and the reporting so far has been highly variable. We suggest using the STROBE/STREGA reporting guidelines for individual studies to allow evaluation of interaction on both scales. The development of new consortia for specific neurolog- ical disorders that include both genetic and environmental data might offer benefits for GxE meta-analyses in the future.

\section{Disclosure Statement}

The authors have nothing to disclose.

\section{References}

1 Hunter DJ: Gene-environment interactions in human diseases. Nat Rev Genet 2005;6: 287-298.

- 2 Lin R, Taylor BV, Simpson S, Jr., Charlesworth J, Ponsonby AL, Pittas F, Dwyer T, van der Mei IA: Novel modulating effects of PKC family genes on the relationship between serum vitamin $\mathrm{D}$ and relapse in multiple sclerosis. J Neurol Neurosurg Psychiatry 2013, in press.

-3 Ottman R: Gene-environment interaction: definitions and study designs. Prev Med 1996; 25:764-770.

-4 Simon KC, van der Mei IA, Munger KL, Ponsonby A, Dickinson J, Dwyer T, Sundstrom P, Ascherio A: Combined effects of smoking, anti-EBNA antibodies, and HLA-DRB1*1501 on multiple sclerosis risk. Neurology 2010;74 1365-1371.

5 Rothman KJ: Synergy and antagonism in cause-effect relationships. Am J Epidemiol 1974; $99: 385-388$

6 Skrondal A: Interaction as departure from additivity in case-control studies: a cautionary note. Am J Epidemiol 2003;158:251-258.

7 Rothman K: Interaction between causes; in Rothman K (ed): Modern Epidemiology. Boston, Little, Brown and Company, 1986, pp 311-326.

8 Botto LD, Khoury MJ: Commentary: facing the challenge of gene-environment interaction: the two-by-four table and beyond. Am J Epidemiol 2001;153:1016-1020.

-9 Piegorsch WW, Weinberg CR, Taylor JA: Non-hierarchical logistic models and caseonly designs for assessing susceptibility in population-based case-control studies. Stat Med 1994;13:153-162.

10 Dennis J, Hawken S, Krewski D, Birkett N, Gheorghe M, Frei J, McKeown-Eyssen G, Little J: Bias in the case-only design applied to studies of gene-environment and gene-gene interaction: a systematic review and metaanalysis. Int J Epidemiol 2011;40:1329-1341.

11 Crabb DW, Matsumoto M, Chang D, You M: Overview of the role of alcohol dehydrogenase and aldehyde dehydrogenase and their variants in the genesis of alcohol-related pathology. Proc Nutr Soc 2004;63:49-63.
Gatto NM, Campbell UB, Rundle AG, Ahsan $\mathrm{H}$ : Further development of the case-only design for assessing gene-environment interaction: evaluation of and adjustment for bias. Int J Epidemiol 2004;33:1014-1024.

13 Wacholder S, Rothman N, Caporaso N: Population stratification in epidemiologic studies of common genetic variants and cancer: quantification of bias. J Nat Cancer Inst 2000; 92:1151-1158.

14 Higgins JPT, Green S (eds): Cochrane Handbook for Systematic Reviews of Interventions, version 5.1.0. The Cochrane Collaboration, updated March 2011. www.cochrane-handbook.org.

15 Altman DG, Bland JM: Interaction revisited: the difference between two estimates. BM] 2003;326:219.

16 Sutton AJ, Higgins JP: Recent developments in meta-analysis. Stat Med 2008;27:625-650.

17 Kim-Cohen J, Caspi A, Taylor A, Williams B, Newcombe R, Craig IW, Moffitt TE: MAOA, maltreatment, and gene-environment interaction predicting children's mental health: new evidence and a meta-analysis. Mol Psychiatry 2006;11:903-913.

18 Thompson SG, Higgins JP: How should metaregression analyses be undertaken and interpreted? Stat Med 2002;21:1559-1573.

19 Peters JL, Sutton AJ, Jones DR, Abrams KR, Rushton L: Comparison of two methods to detect publication bias in meta-analysis. JAMA 2006;295:676-680.

20 Minelli C, Thompson JR, Abrams KR, Thakkinstian A, Attia J: The quality of meta-analyses of genetic association studies: a review with recommendations. Am J Epidemiol 2009; 170:1333-1343.

21 Little J, Higgins JPT (eds): The HuGENet HuGE Review Handbook, version 1.0. 2006. http://www.hugenet.ca.

22 Palla L, Higgins JP, Wareham NJ, Sharp SJ: Challenges in the use of literature-based meta-analysis to examine gene-environment interactions. Am J Epidemiol 2010;171:12251232.
23 Keijzer MB, Borm GF, Blom HJ, Bos GM Rosendaal FR, den Heijer M: No interaction between factor $\mathrm{V}$ Leiden and hyperhomocysteinemia or MTHFR 677TT genotype in venous thrombosis: results of a meta-analysis of published studies and a large case-only study. Thromb Haemost 2007;97:32-37.

24 Zeiger JS, Beaty TH, Liang KY: Oral clefts, maternal smoking, and TGFA: a meta-analysis of gene-environment interaction. Cleft Palate Craniofac J 2005;42:58-63.

25 Kocarnik JD, Hutter CM, Slattery ML, Berndt SI, Hsu L, Duggan DJ, Muehling J, Caan BJ, Beresford SA, Rajkovic A, Sarto GE, Marshall JR, Hammad N, Wallace RB, Makar KW, Prentice RL, Potter JD, Hayes RB, Peters U: Characterization of 9 p24 risk locus and colorectal adenoma and cancer: gene-environment interaction and meta-analysis. Cancer Epidemiol Biomarkers Prev 2010;19: 3131-3139.

26 Marcus PM, Hayes RB, Vineis P, Garcia-Closas M, Caporaso NE, Autrup H, Branch RA, Brockmoller J, Ishizaki T, Karakaya AE, Ladero JM, Mommsen S, Okkels H, Romkes M, Roots I, Rothman N: Cigarette smoking, Nacetyltransferase 2 acetylation status, and bladder cancer risk: a case-series meta-analysis of a gene-environment interaction. Cancer Epidemiol Biomarkers Prev 2000;9:461-467.

27 Hartz SM, Short SE, Saccone NL, et al: Increased genetic vulnerability to smoking at CHRNA5 in early-onset smokers. Arch Gen Psychiatry 2012;69:854-860

28 Nettleton JA, Hivert MF, Lemaitre RN, et al: Meta-analysis investigating associations between healthy diet and fasting glucose and insulin levels and modification by loci associated with glucose homeostasis in data from 15 cohorts. Am J Epidemiol 2013;177:103-115.

29 Stang A: Critical evaluation of the NewcastleOttawa scale for the assessment of the quality of nonrandomized studies in meta-analyses. Eur J Epidemiol 2010;25:603-605.

30 Morimoto LM, White E, Newcomb PA: Selection bias in the assessment of gene-environment interaction in case-control studies. Am J Epidemiol 2003;158:259-263. 
-31 Wacholder S, Chatterjee N, Hartge P: Joint effect of genes and environment distorted by selection biases: implications for hospitalbased case-control studies. Cancer Epidemiol Biomarkers Prev 2002;11:885-889.

32 Clayton D, McKeigue PM: Epidemiological methods for studying genes and environmental factors in complex diseases. Lancet 2001; 358:1356-1360.

33 Garcia-Closas M, Lubin JH: Power and sample size calculations in case-control studies of gene-environment interactions: comments on different approaches. Am J Epidemiol 1999;149:689-692.

34 Ioannidis JP, Ntzani EE, Trikalinos TA: 'Racial' differences in genetic effects for complex diseases. Nat Genet 2004;36:1312-1318.

35 Freedman ML, Reich D, Penney KL, McDonald GJ, Mignault AA, Patterson N, Gabriel SB, Topol EJ, Smoller JW, Pato CN, Pato MT, Petryshen TL, Kolonel LN, Lander ES, Sklar P, Henderson B, Hirschhorn JN, Altshuler D:
Assessing the impact of population stratification on genetic association studies. Nat Genet 2004;36:388-393.

36 Knol MJ, Egger M, Scott P, Geerlings MI, Vandenbroucke JP: When one depends on the other: reporting of interaction in casecontrol and cohort studies. Epidemiology 2009;20:161-166

37 Vandenbroucke JP, von Elm E, Altman DG, Gotzsche PC, Mulrow CD, Pocock SJ, Poole C, Schlesselman JJ, Egger M: Strengthening the Reporting of Observational Studies in Epidemiology (STROBE): explanation and elaboration. PLoS Med 2007;4:e297.

38 Little J, Higgins JP, Ioannidis JP, Moher D, Gagnon F, von Elm E, Khoury MJ, Cohen B, Davey-Smith G, Grimshaw J, Scheet P, Gwinn M, Williamson RE, Zou GY, Hutchings K, Johnson CY, Tait V, Wiens M, Golding J, van Duijn C, McLaughlin J, Paterson A, Wells G, Fortier I, Freedman M, Zecevic M, King R, Infante-Rivard C, Stewart A, Birkett N:
Strengthening the reporting of genetic association studies (STREGA): an extension of the strobe statement. Hum Genet 2009;125:131151.

39 Caspi A, McClay J, Moffitt TE, et al: Role of genotype in the cycle of violence in maltreated children. Science 2002;297:851-854.

40 Foley DL, Eaves LJ, Wormley B, et al: Childhood adversity, monoamine oxidase A genotype, and risk for conduct disorder. Arch Gen Psychiatry 2004;61:738-744.

41 Haberstick BC, Lessem JM, Hopfer CJ, et al: Monoamine oxidase A (MAOA) and antisocial behaviors in the presence of childhood and adolescent maltreatment. Am J Med Genet B Neuropsychiatr Genet 2005;135B: 59-64.

42 Nilsson KW, Sjöberg RL, Damberg M, et al: Role of monoamine oxidase A genotype and psychosocial factors in male adolescent criminal activity. Biol Psychiatry 2006;59:121127 